\title{
Rocuronium bromide induced anaphylaxis in a child -A case report-
}

\author{
Young Ho Jang ${ }^{1}$, Sang Gyu Kim², Yong Hoon Son², and Joon Mo Park² \\ Departments of ${ }^{1}$ Anesthesiology and Pain Medicine, ${ }^{2}$ General Surgery, Pureun Hospital, Daegu, Korea
}

Anaphylaxis or anaphylactoid reaction in pediatric patient during anesthesia is rare. We report a rocuronium induced anaphylactic reaction in a 33-month-old female. The patient was scheduled to undergo escharectomy due to injuries suffered from a major burn. Shortly after administration of rocuronium, the patient developed severe hypotension, tachycardia, and hypoxia. A similar reaction occurred after administration of rocuronium on subsequent anesthesia. She underwent uneventful anesthesia with volatile induction and maintenance of anesthesia with sevoflurane on her next 7 operations without using of muscle relaxant. (Korean J Anesthesiol 2010; 59: 411-415)

Key Words: Anaphylactoid reaction, Anaphylaxis, Muscle relaxant, Pediatrics, Rocuronium.

Hypersensitivity reactions that might occur during anesthesia, i.e., anaphylaxis and anaphylactoid reaction, have been reported to have a variable degree of the incidence depending on the authors. But it has been reported to be approximately $1 / 3,000-20,000[1,2]$ and its mortality has been reported to be $3-6 \%$ [1]. Of the drugs which are used for anesthesia, muscle relaxants have the highest frequency of inducing anaphylaxis. In addition, such substances as latex or antibiotics can also induce the occurrence of anaphylaxis [3]. On the other hand, the occurrence of anaphylaxis due to anesthetic agents has been frequently reported in men in their fifties and women in their forties. In pediatric patients aged ten years or younger, it occurs to such a rare extent as $<4 \%$ of total cases of anaphylaxis [3].

In the mid-1990s, rocuronium bromide (one of the nondepolarizing aminosteroid muscle relaxants with a short action time) has been introduced in a clinical setting. As described here, according to the increased use of rocuronium, the occurrence of rocuronium anaphylaxis has also been reported to rise $[1,4]$. Also in Korea, two cases of rocuronium anaphylaxis have been reported up to present $[5,6]$. But most of these cases have been reported to occur in middle-aged and elderly people. We experienced a case of anaphylaxis occurring due to rocuronium during anesthesia in a 33-month-old pediatric patient. To our knowledge, this is the youngest pediatric case of rocuroniuminduced anaphylaxis.

\section{Case Report}

A 33-month-old female weighed $15 \mathrm{~kg}$ visited us with chief complaints of deep flame burn with a $38 \%$ body surface area of grade 2 and 3 in such areas as face, body trunk, and both arms. The patient had no past history of recent upper respiratory

Received: July 20, 2009. Revised: 1st, August 30, 2009; 2nd, September 20, 2009. Accepted: September 21, 2009.

Corresponding author: Young Ho Jang, M.D., Ph.D., Department of Anesthesiology and Pain Medicine, Pureun Hospital, 2033-13, Daemyung 2-dong, Nam-gu, Daegu 705-821, Korea. Tel: 82-53-471-2800, Fax: 82-53-471-2806, E-mail: weonjo@dsmc.or.kr

(c) This is an open-access article distributed under the terms of the Creative Commons Attribution Non-Commercial License (http:// creativecommons.org/licenses/by-nc/3.0/), which permits unrestricted non-commercial use, distribution, and reproduction in any medium, provided the original work is properly cited. 
tract infection, asthma, atopy, and allergic reactions to foods or drugs. On physical examination, the patient had no notable findings other than burn injury. On admission, the patient was transferred to an operating room for emergency care and then intravenously given thiopental sodium $80 \mathrm{mg}$. The patient received manual ventilation using $\mathrm{N}_{2} \mathrm{O} / \mathrm{O}_{2}$ and sevoflurane. Without the administration of muscle relaxants, the patient underwent endotracheal intubation using an endotracheal tube with an inner diameter of $4.0 \mathrm{~mm}$. Then, the patient underwent central venous, arterial, and urinary catheterization. This was followed by wound disinfection and dressing. At this time, the total anesthetic time was 60 minutes. The systolic and diastolic pressures were 90-100 $\mathrm{mmHg}$ and $45-60 \mathrm{mmHg}$, respectively, and the heart rate was $110-135$ beats/min. Pulse oxymetry saturation $\left(\mathrm{SPO}_{2}\right)$ was maintained at $100 \%$. Thereafter, the patient was transferred to an intensive care unit (ICU) while having an endotracheal tube removed following a recovery of the mental alertness. Then, the patient underwent an intensive care through wound disinfection and fluid therapy.

Three days later, the patient was supposed to escharectomy and cadaver skin graft under general anesthesia and then transferred to an operating room. The preoperative vital signs and routine laboratory results were within normal limits. To induce the anesthesia, following the pure oxygenation, propofol $30 \mathrm{mg}$ and rocuronium bromide $10 \mathrm{mg}$ (Esmeron, Hanhwa Pharmaceuticals Corp., Korea) were intravenously injected. Approximately 1 minute after the administration of rocuronium, i.e., immediately before the endotracheal intubation, the resistance was perceived at a reservoir bag. Meanwhile, $\mathrm{SPO}_{2}$ was abruptly decreased up to $84 \%$. The heart rate was increased up to 170 beats/min. This was followed by an endotracheal intubation. At this time, the systolic pressure by an arterial catheter was decreased to 50-60 $\mathrm{mmHg}$. On auscultation, the secretion sound was heard from the lung. The airway pressure was increased up to $30 \mathrm{cmH}_{2} \mathrm{O}$, based on which the abnormal location of an endotracheal tube or its obstruction was suspected. Following removal of endotracheal tube, the endotracheal intubation was performed again with the use of an endotracheal tube with an inner diameter of 4.5 $\mathrm{mm}$. To increase the blood pressure, the fluid was promptly administered at a rate of $150 \mathrm{ml} / \mathrm{hr}$. Meanwhile, 100\% oxygen was also administered and ephedrine $4 \mathrm{mg}$ was administered twice. Following this, the systolic blood pressure was elevated to $110 \mathrm{mmHg}$, however, hypoxia and a tachycardia of $>170$ beats/ min were continued. On arterial blood gas analysis, there were such measurements as pH 7.223, $\mathrm{PCO}_{2} 51.9 \mathrm{mmHg}, \mathrm{PO}_{2} 75.2$ $\mathrm{mmHg}$ and $\mathrm{BE}-7.1 \mathrm{mmol} / \mathrm{L}$. The patient was transferred to ICU and the mechanical ventilation was performed by using midazolam and vecuronium for sedation. As soon as the patient reached ICU, the patient received a chest X-ray. According to this, the patient had findings suggestive of the pulmonary edema accompanying a shadow in both lungs (Fig. 1). Three days following an ICU treatment, the patient was found to have improved pulmonary edema. Then, the patient was transferred to an operation room in order to take the planned surgery in such a condition that an endotracheal tube was left. For anesthesia, the spontaneous respiration was maintained using $\mathrm{N}_{2} \mathrm{O} / \mathrm{O}_{2}$ and sevoflurane with intermittent manually assisted ventilation. During the escharectomy on the abdominal area, the abdominal mobility due to a spontaneous respiration was severe. For the relaxation of abdominal msucles, the patient received an intravenous injection of rocuronium $10 \mathrm{mg}$. Approximately 2 minutes following an intravenous injection of rocuronium, without the abnormality of respiratory circuit, the airway pressure was increased. Meanwhile, $\mathrm{SPO}_{2}$ was decreased up to $75 \%$. The systolic pressure was decreased to $40-50 \mathrm{mmHg}$. On arterial blood gas analysis, there were such measurements as pH 7.351, $\mathrm{PCO}_{2} 41.1 \mathrm{mmHg}, \mathrm{PO}_{2} 49.7 \mathrm{mmHg}$ and $\mathrm{BE}-3.1$ $\mathrm{mmol} / \mathrm{L}$. Along with the administration of fluids, ephedrine 4 mg was intravenously injected twice. With the maintenance of
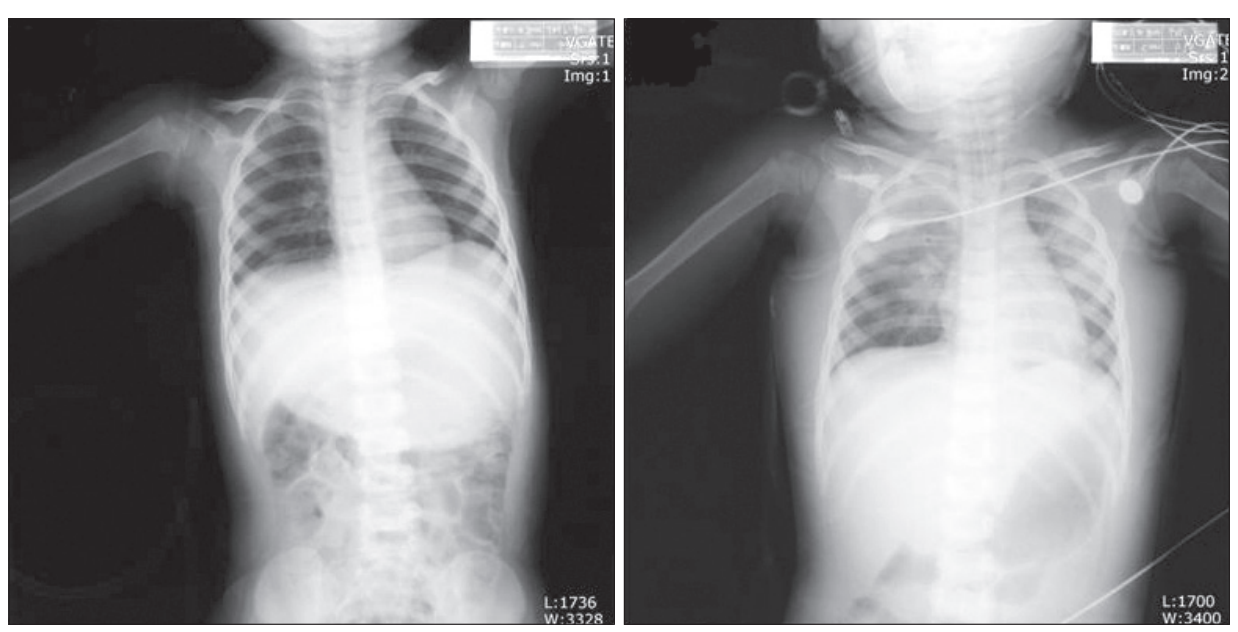

Fig. 1. Preoperative chest X-ray reveals unremarkable (left). Chest X-ray taken immediately after hypersensitivity reaction showes pulmonary edema with illdefined hazziness at both lung, especially perihilar area, and accentuation of bronchovascular markings at both lung, especially perihilar area (right). 
anesthesia using sevoflurane, $\mathrm{N}_{2} \mathrm{O}$ was discontinued and $100 \%$ oxygen was administered. Hypotension and hypoxia $\left(\mathrm{SPO}_{2}\right.$ : approximately $75 \%$ ) were persistently present. Accordingly, 1 : 1,000 epinephrine $0.15 \mathrm{mg}$ was intramuscularly administered. Thereafter, the tachycardia was persistently present. Besides, the blood pressure and $\mathrm{SPO}_{2}$ were recovered. At this time, there were such measurements as $\mathrm{pH} 7.329, \mathrm{PCO}_{2} 42.9 \mathrm{mmHg}, \mathrm{PO}_{2}$ $124.3 \mathrm{mmHg}$ and $\mathrm{BE}-3.9 \mathrm{mmol} / \mathrm{L}$. A surgery was performed as planned. Intraoperatively, the cumulative packed red cells were administered at a volume of $80 \mathrm{ml}$. The total anesthetic time was 115 minutes. Immediately before a surgery was completed, dexamethasone $0.5 \mathrm{mg}$ was administered. With an intravenous injection of neostigmine $1 \mathrm{mg}$ and atropine $0.2 \mathrm{mg}$, the spontaneous respiration was recovered. Following the removal of an endotracheal tube, the recovery of mental alertness was confirmed. Then, the patient was transferred to ICU. Thirteen days later, the secondary escharectomy was planned. At this time, without the administration of muscle relaxants, the anesthesia was induced using propofol. Then, with the use of $\mathrm{N}_{2} \mathrm{O} / \mathrm{O}_{2}$ and sevoflurane, the anesthesia was maintained. Thereafter, after the additional escharectomy was performed six times with volatile induction and maintenance of anesthesia with sevoflurane without muscle relaxants, skin graft was done. Two months and the extra ten days following an outpatient visit, the patient was discharged without notable complications.

\section{Discussion}

We experienced a case in which anaphylaxis was strongly suspected based on the findings that hypotension, tachycardia and hypoxia due to the use of rocuronium which was used for general anesthesia twice in a 33-month-old pediatric patient. Two types of immediate hypersensitivity reactions including anaphylaxis and anaphylactoid reaction are classified according to the involvement of such antibodies as immuoglobulin E (IgE). But it is not easy to make a differential diagnosis of these two reactions based on the clinical symptoms. Instead, they can be classified according to skin test or biologic test. In association with this, Mertes et al. [7] reported that it would be desirable to use such terms as anaphylactoid reaction in cases in which a diagnosis of immunological mechanisms was not made through an allergic test. In the present case, parents refused to take skin test for the patient. Therefore, due to a lack of the immediate biological test, the current case would correspond to the anaphylactoid reaction.

In regard to most cases of the allergy occurring during anesthesia, i.e., acute hypersensitivity reaction, grade-2 responses (non-life-threatening skin reaction, hypotension accompanying tachycardia, coughing or the difficulty of mechanical respiration) or grade-3 responses (life-threatening cardiovascular collapse, bradycardia or tachycardia and severe bronchospasm) account for approximately $85 \%$ of total cases [3]. The current case is the one that is suspected to be grade- 2 or grade- 3 anaphylaxis occurring due to rocuronium in a 33-month-old pediatric patient. To make an accurate diagnosis of anaphylaxis or anaphylactoid reaction, in addition to clinical symptoms, the concentrations of tryptase, histmaine or IgE which are present in the blood should be measured. Otherwise, after several weeks following the occurrence of allergic reactions, skin prick test or intradermal test should be performed $[1,8]$. Skin reactions occurring during the allergic responses due to drugs may be manifested as urticaria, edema, pruritis, thermal sensation and red spots. In the current case, however, these skin reactions were not perceived because of a burn injury. In addition, in the current case, at the time of onset of symptoms, the life saving treatment of child was considered to be important. Accordingly, the blood test for anaphylaxis could not be performed. However, at two anesthetic sessions, tachycardia, hypotension and hypoxia occurred identically within 2 minutes immediately after rocuronium was administered. Thereafter, during the other seven sessions of anesthesia for which rocuronium was not used, these symptoms did not occur. In this regard, anaphylaxis due to rocuronium could be powerfully suspected.

On the other hand, propofol may also cause the occurrence of anaphylaxis $[9,10]$. In the first onset of case, propofol and rocuronium were concurrently used. In the second onset of case, without using propofol, rocuronium was solely used. Besides, thereafter, in the anesthetic sessions where propofol was used but rocuronium was not used, these responses did not occur. Based on these findings, in the current case, the possibility for anaphylaxis due to propofol could be ruled out.

Anaphylaxis occurring during anesthesia occurs at a frequency which is approximately four times higher in men than women [8]. In regard to the age, it occurs the most frequently in people in their forties. The occurrence in pediatric patients aged ten years or younger has been rarely reported at a frequency which is lower than $4 \%$ of total cases of anaphylaxis [3]. Muscle relaxants are the most common drugs that cause the occurrence of anaphylaxis to a life-threatening extent during anesthesia, which account for approximately $50-70 \%$ of causes which are responsible for anaphylaxis occurring during ansethesia [11,12]. According to the reports made by Laxenaire et al. [3] about anaphylaxis which occurred during anesthesia for two years, anaphylaxis occurring due to muscle relaxants occurred the most frequently in people aged ten years or younger. Besides, it also occurred the most frequently in women aged in their thirties. In cases of rocuronium, however, there are extremely rare cases in which anaphylaxis occurred in a 33-monthold pediatric patient as shown in the present case. Clinical 
characteristics due to rocuronium are mainly classified into two types: cardiovascular collapse and bronchospasm [1]. Generally in cases in which bronchospasm occurred due to anaphylaxis, due to the actions of alpha-1 elevating the blood pressure and those of beta- 2 relaxing the bronchial smooth muscles, the immediate administration of epinephrine is important. Besides, in cases of cardiovascular collapse due to anaphylaxis, fluid supply is generally done and epinephrine and phenylephrine are administered. In cases which are refractory to these treatments, the administration of vasopressin could be of help [5]. Hypotension due to anaphylaxis cannot be well responded to ephedrine. In the current case, in the first onset of symptoms, the resistance was perceived during manual ventilation using a mask prior to endotracheal intubation. This indicates that there is a possibility for respiratory tract obstruction such as the upper airway obstruction, laryngospasm or bronchospasm. Thereafter, immediately after an endotracheal intubation was performed, the respiratory tract pressure was increased. The ventilation accompanying the mobility of chest was possible. It is therefore assumed that there is a remote possibility for the occurrence of bronchospasm. On the other hand, in the second onset of anaphylaxis, in such a condition that an endotracheal tube was placed, hypotension and increase in airway pressure occurred during surgery. Based on these findings, the possibility for bronchospasm is suspected. This is immediately responded to epinephrine.

Due to the increased permeability of capillary during the occurrence of anaphylaxis, the plasma volume is abruptly increased. A massive amount of fluid therapy might also be essential in this case. Owing to this, the edema might occur to the lung or respiratory tract. Also in the current case, following the first onset of anaphylaxis, on chest radiography which was performed in ICU setting, there was a finding that is suggestive of pulmonary edema. This might be due to the increased permeability of pulmonary capillary vessels because of anaphylaxis. Pulmonary edema occurring in the current case was recovered to the normal status after two days. But we assumed the possibility that pulmonary edema might originate from the negative pressure of respiratory tract. At the time, we could not speculate the possibility for anaphylaxis due to rocuronium.

On the other hand, rocuronium is an amino-steroid nondepolarizing muscle relaxant whose pharmacological characteristics are similar to those of vecuronium. In cases of allergic reactions due to rocuronium, there might be crossreactions with other types of steroid non-depolarizing muscle relaxants. In particular, aminosteroid muscle relaxants have a higher prevalence of the cross-reactions as compared with benzilisoquinoline-derived muscle relaxants [13]. These muscle relaxants commonly have tetra-positively charged ammonium ion $\left(\mathrm{NH}_{4}^{+}\right)$as an antigenic determinant. Due to this chemical structure, the cross-reaction can occur. This should therefore be confirmed [14]. In the current case, however, the anaphylaxis occurred due to rocuronium during anesthesia. Following the use of vecuronium which was used for mechanical ventilation at an ICU, however, no anaphylaxis occurred. Accordingly, the possibility for cross-reactions between the two drugs was ruled out in the present case.

Generally in pediatric patients, anaphylaxis rarely occurs during anesthesia. We experienced a case in which rocuroniuminduced grade- 2 or grade- 3 anaphylaxis was strongly suspected following two sessions of general anesthesia in a 33-monthold pediatric patient. Although the occurrence of rocuroniuminduced anaphylaxis has been reported more frequently than that due to vecuronium or pancuronium and less frequently than that due to succinylcholine [8], there is a tendency that the use of succinylcholine has recently been decreased and that of rocuronium has been increased. Owing to this, the occurrence of rocuronium-induced anaphylaxis may be increased. On the other hand, the use of muscle relaxants can make it easier to perform an endotracheal intubation and it can also reduce the demand on major anesthetic agents. It is also advantageous that the postoperative complications associated with the respiratory tract can be reduced. But anesthesiologists can consider the methods for reducing risks of developing fatal anaphylaxis due to muscle relaxants by performing a laryngeal mask or an endotracheal intubation without the administration of muscle relaxants such as rocuronium.

\section{References}

1. Heier T, Guttormsen AB. Anaphylactic reactions during induction of anaesthesia using rocuronium for muscle relaxation: a report including 3 cases. Acta Anaesthesiol Scand 2000; 44: 775-81.

2. Mertes PM, Aimone-Gastin I, Guéant-Rodriguez RM, MoutonFaivre C, Audibert G, O'Brien J, et al. Hypersensitivity reactions to neuromuscular blocking agents. Curr Pharm Des 2008; 14: 2809-25.

3. Laxenaire MC, Mertes PM; Groupe d'Etudes des Réactions Anaphylactoïdes Peranesthésiques. Anaphylaxis during anaesthesia: results of a two-year survey in France. Br J Anaesth 2001; 87: 549-58.

4. Meng L, Williams EL. Case report: treatment of rocuroniuminduced anaphylactic shock with vasopressin. Can J Anaesth 2008; 55: 437-40.

5. Lee HM, Song SO. Anaphylaxis after injection of rocuronium: a case report. Korean J Anesthesiol 2006; 51: 101-4.

6. Hwang SM, Suh JW, Lim SY. Anaphylaxis after thiopental and rocuronium Induction: a case report. Korean J Anesthesiol 2005; 48: 95-8.

7. Mertes PM, Laxenaire MC, Alla F; Groupe d'Etudes des Réactions Anaphylactoïdes Peranesthésiques. Anaphylactic and anaphylactoid reactions occurring during anesthesia in France in 1999-2000. Anesthesiology 2003; 99: 536-45. 
8. Rose M, Fisher M. Rocuronium: high risk for anaphylaxis? Br J Anaesth 2001; 86: 678-82.

9. Laxenaire MC, Mata-Bermejo E, Moneret-Vautrin DA, Gueant JL. Life-threatening anaphylactoid reactions to propofol (Diprivan). Anesthesiology 1992; 77: 275-80.

10. McNeill O, Kerridge RK, Boyle MJ. Review of procedures for investigation of anaesthesia-associated anaphylaxis in Newcastle, Australia. Anaesth Intensive Care 2008; 36: 201-7.

11. Mertes PM, Laxenaire MC. Allergy and anaphylaxis in anaesthesia. Minerva Anestesiol 2004; 70: 285-91.
12. Watkins J. Adverse reaction to neuromuscular blockers: frequency, investigation, and epidemiology. Acta Anaesthesiol Scand Suppl 1994; 102: 6-10.

13. Laxenaire MC, Gastin I, Moneret-Vautrin DA, Widmer S, Guéant JL. Cross-reactivity of rocuronium with other neuromuscular blocking agents. Eur J Anaesthesiol Suppl 1995; 11: 55-64.

14. Matthey P, Wang P, Finegan BA, Donnelly M. Rocuronium anaphylaxis and multiple neuromuscular blocking drug sensitivities. Can J Anaesth 2000; 47: 890-3. 